\title{
Hidden hassium
}

\author{
From its scarcity to political intrigue over naming conventions, element 108's story describes how international \\ cooperation overcame the limits of nuclear science, says Michael Tarselli.
}

$\Lambda$ casual aside uttered by Nottingham Professor Sir Martyn Poliakoff during his Periodic Table of Videos series (http://www.periodicvideos.com/videos/108. htm) reflects a general feeling: "Hassium ... I know nothing about hassium. Shall we make something up?". Among the already little-known super-heavies, element 108 doesn't have the controlled reactivity of a seaborgium atom, or the relative stability of fermium - the longest-lived isotope is ${ }^{257} \mathrm{Fm}$ with a net 100 day half-life, and ${ }^{252} \mathrm{Fm}$ is believed to resist spontaneous fission rather well ${ }^{1}$, though it undergoes $\alpha$-decay. In contrast the half-life of hassium's most stable isotope, ${ }^{270} \mathrm{Hs}$, is only a few seconds. Estimates for the total number of hassium atoms created so far run from a few dozen to as many as 100 atoms. Ever.

How were those lone atoms produced? Let's rewind to 1984 . Major nuclear research was emerging from three countries, two of which had outmoded names: West Germany, the USSR and the USA. Back then, element 108 was simply known as Uno for 'unniloctium', literally meaning 'one-zero-eight' according to a system of numerical roots adopted in $1979^{2}$. Since the 1940s, scientists had prepared novel transactinides by bombarding uranium with neutron beams. This approach, however, only works up to element 100 (fermium). The team at the Joint Institute for Nuclear Research (JINR) in Dubna, USSR (now Russia), led by actinide heavyweight Yuri Oganessian, then pioneered the techniques of 'cold' fusion (collisions of two early elements such as iron and bismuth) and 'hot' fusion (using actinide radionuclides as the targets).

In hot fusion, researchers bombard a heavy target such as einsteinium or plutonium with a beam of lighter elements such as carbon or oxygen. This early technique, according to Peter Armbruster ${ }^{3}$ from Germany's GSI Helmholtz Centre for Heavy Ion Research, worked fine up to element 106 (seaborgium). New equipment later allowed beams of heavier nuclei such as

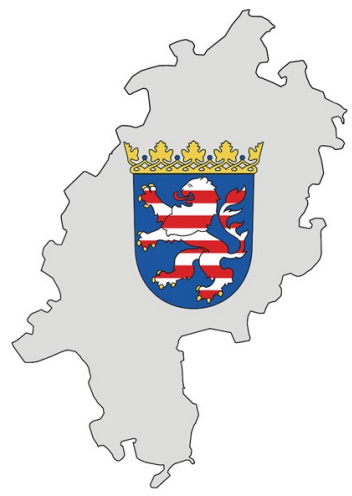

Credit: michal812 / Alamy Stock Photo

calcium or iron to be fired, pushing back the limit to 118 ...so far. The first hassium atoms were synthesized by bombarding a lead target with iron atoms. The process was later optimized, and firing ${ }^{26} \mathrm{Mg}$ at a target made of ${ }^{248} \mathrm{Cm}$ gave ${ }^{270} \mathrm{Hs}$ - which was dubbed the doubly magic isotope ${ }^{4}$.

Along with new elements came numerous priority disagreements, and thus naming controversies - so much so that the International Unions of Pure and Applied Chemistry and Physics (IUPAC and IUPAP) created the Transfermium Working Group, in which scientists from the three main heavyelement-discovering nations first oversaw the recognition process for elements 101-109. In 1994, this group advanced the names hahnium and meitnerium for elements 108 and 109, for nuclear fission discoverers Otto Hahn and Lise Meitner, respectively. Meitnerium was adopted, but for 108 the suggestion of the German team, led by Peter Armbruster and Gottfried Münzenberg, was the one ratified in 1997: hassium, which honours the German state of Hesse (whose map and coat of arms are pictured).

Perhaps the most tantalizing aspect of hassium is that it's nearly unexplored. We still don't know many of its physical properties - melting point, boiling point, pressure or heat capacity. Looking at its group 8 cousins iron, ruthenium and osmium one expects it might be a solid, but this is tough to tell from a handful of atoms stuck to a silicon detector. When you have only traces of a specific element, how do you investigate its properties? Invent some fairly specialized lab equipment. Hassium is tough to separate away from other superheavies and by-products of the fusion reaction; specialized detectors were built to study its characteristic $\alpha$-decay and probe its reaction chemistry. In 2002 the GSI, JINR, the Lawrence Berkeley National Laboratory and seven other institutions joined forces to create seven atoms of hassium. Those were then pushed through a stream of oxygen to yield a highly volatile oxide with similar properties to ruthenium and osmium tetroxides - "presumably $\mathrm{HsO}_{4}$ ", whose single ${ }^{269} \mathrm{Hs}$ atom was identified by $\alpha$-decay ${ }^{5}$

In a 2011 Science Perspective ${ }^{6}$, nuclear physicist Walter Greiner wryly suggested that neutron-rich nuclei, such as hassium, might be feasibly made by setting off "two or three nuclear explosions near a suitably protected target buried deep underground.' As multiple global treaties prohibit this, we will have to keep relying on technologies such as bombarding accelerator targets with neutron-rich beams of ${ }^{48} \mathrm{Ca}$ or ${ }^{58} \mathrm{Fe}$. Even if we have to wait a few more decades for the next generation of nuclear scientists to produce enough hassium to fill in the blank spaces of our knowledge, it's probably for the best.

Michael A. Tarselli

NIBR Informatics (NX), Novartis Institutes for BioMedical Research, Cambridge, MA, USA.

e-mail:mike.tarselli@novartis.com

Published online: 22 March 2018

https://doi.org/10.1038/s41557-018-0037-4

References

1. Staszczak, A., Baran, A. \& Nazarewicz, W. Preprint at https://arxiv.org/abs/1208.1215 (2012).

2. Chatt, J. Pure Appl. Chem. 51, 381-384 (1979).

3. Armbruster, P. \& Hessberger, F. P. Making New Elements. Scientific American (September 1998); http://go.nature.com/2GWiHyG

4. Dvorak, J. et al. Phys. Rev. Lett. 97, 242501 (2006).

5. Düllmann, Ch. E. et al. Nature 418, 859-862 (2002).

6. Clery, D. Science 333, 1377-1379 (2011). 